ISBN 978-981-14-1684-2

Proceedings of 2019 the 9th International Workshop on Computer Science and Engineering

(WCSE 2019 SUMMER)

Hong Kong, 15-17 June, 2019, pp. 201-206

doi: $10.18178 /$ wcse.2019.06.030

\title{
Research on Swing up and Stabilization of the Single Rotating Inverted Pendulum Based on LabVIEW
}

\author{
Li Xinqi ${ }^{1,2,+}$, Zhang Yongli ${ }^{1,2}$, Cui Shigang ${ }^{1,2}$ and Liu Yu ${ }^{1,2}$ \\ 1 School of Automation and Electrical Engineering, Tianjin University of Technology and Education, Tianjin \\ ${ }^{2}$ Tianjin Key Laboratory of Information Sensing and Intelligent Control, Tianjin 300222, China

\begin{abstract}
The inverted pendulum system is a typical nonlinear, multivariable, and strong coupling system. In this paper, the structure of the inverted pendulum is introduced in detail. The inverted pendulum produced by Quanser Company of Canada is taken as the research object. Based on the LabVIEW software, the inverted pendulum can be swing up automatically by energy method. The result suggests that the regulator designed in this article shows great control.
\end{abstract}

Keywords: Single Inverted Pendulum, Swing-up Control, Linear Quadratic Regulator

\section{Introduction}

LabVIEW is a graphical programming language developed by National Instruments (NI) [1]. It is widely used in by industry, academia and research laboratories and is regarded as a standard data collection and instrument control software with features such as visualization and easy programming.

The inverted pendulum system is a typical nonlinear, multivariable, order and strong coupling system [24]. Since the inverted pendulum system can express many abstract system control concepts such as system stability and control ability, the techniques and methods produced by the inverted pendulum system are widely used in artificial intelligence, robot control, missile interception, And other fields .

The study of the inverted pendulum system consists of two parts: the swing up control and the stable control of the equilibrium position[5]. On the basis of drawing on the advanced scientific research results at home and abroad, the single inverted pendulum produced by Canadian company Quanser was taken as the research object. Based on the LabVIEW software, the energy method was selected to complete the system swing control, and the transient and robust performance were selected. A good linear quadratic optimal controller implemented the stable control of the system. Experiments showed that this method can achieve the swing-up control of the single inverted pendulum and has good control effect.

\section{Design of Control System}

\subsection{Control system structure for inverted pendulum}

When ignoring air resistance and various frictional forces, the inverted pendulum system body can be abstracted into a rigid body system consisting of a homogeneous mass, a connecting rod and a pendulum. Let the two generalized coordinates of the system be $\theta_{1}, \theta_{2}$, respectively. The schematic diagram of the system mathematical structure is shown in the fig 1 .

According to Lagrange modeling method, the nonlinear mathematical model of the system can be obtained ${ }^{[6]}$.

$$
M \ddot{q}+N=F_{q}
$$

${ }^{+}$Corresponding author Tel.: + 13622133013;

E-mail address: $184523662 @$ qq.com 
where $M=\left\lfloor\begin{array}{cc}J_{1}+m_{1} l_{1}^{2}+m_{2} l_{2}^{2} \sin ^{2} \theta_{2} & 2 m_{2} l_{1} l_{2} \cos \theta_{2} \\ 2 m_{2} l_{1} l_{2} \cos \theta_{2} & J_{2}+m_{2} l_{2}^{2}\end{array}\right\rfloor N=\left[\begin{array}{c}-2 m_{2} l_{1} l_{2} \sin \theta_{2} \dot{\theta}_{2}^{2}+2 m_{2} l_{2}^{2} \dot{\theta}_{2} \dot{\theta}_{1} \sin \theta_{2} \cos \theta_{2} \\ -m_{2} l_{2}^{2} \dot{\theta}_{1}^{2} \sin \theta_{2} \cos \theta_{2}-m_{2} g l_{2} \sin \theta_{2}\end{array}\right], \quad F_{q}=\left\lfloor\begin{array}{l}u \\ 0\end{array}\right\rfloor$ $J_{l}$ is the moment of inertia of the connecting rod around the $\mathrm{Z}$ axis : $J_{1}=\frac{1}{3} m_{1} l_{1}^{2} ; J_{2}$ is the moment of inertia for a pendulum to rotate around its axis: $J_{2}=\frac{1}{3} m_{2} l_{2}^{2}$.

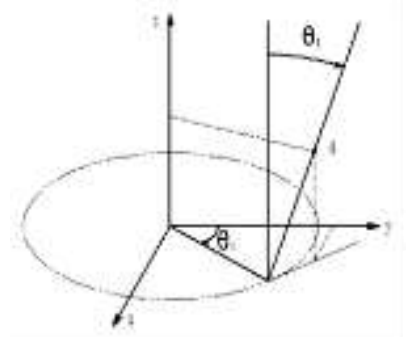

Fig. 1: Simplified mathematical model of system.

The single inverted pendulum hardware system consists of three parts: the inverted pendulum body, the control system and the control platform. This experiment used NI ELVIS II as the control system, read the angle encoder input through the module, and transmitted the data to the computer through the USB interface to provide commands for the power amplifier to drive the DC motor. Use LabVIEW to build a control platform to realize the role of real-time display data and control system. The working principle of the control system is shown in Figure 2.

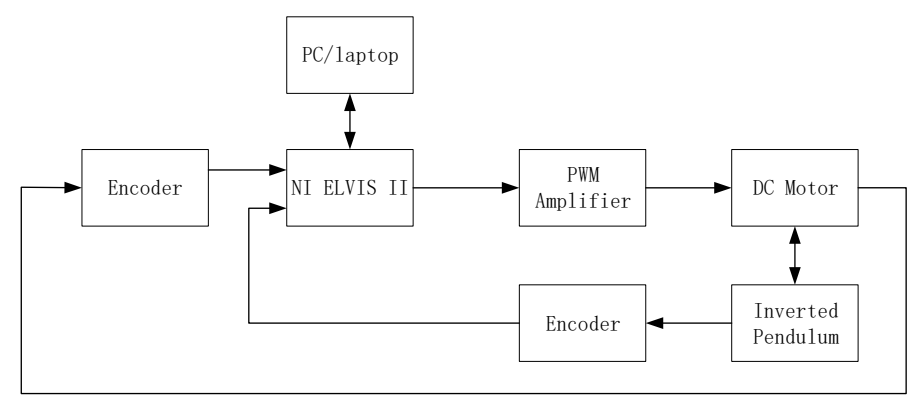

Fig. 2: Working principle diagram of control system.

\subsection{The inverted pendulum swing control based on energy method}

The swinging process of the annular inverted pendulum system can be expressed in the following system characteristic equation

$$
\left[\begin{array}{c}
q \\
\dot{q}
\end{array}\right]=\left\lfloor\begin{array}{l}
0 \\
0 \\
0 \\
0
\end{array}\right\rfloor \rightarrow\left[\begin{array}{l}
q \\
\dot{q}
\end{array}\right]=\left\lfloor\begin{array}{l}
0 \\
\pi \\
0 \\
0
\end{array}\right\rfloor
$$

or Ep $\rightarrow E p 0$. If the arm angle of the system remains constant and the initial position of the pendulum bar is known, the system will oscillate back and forth at constant amplitude. The coordinate system is established by taking the rotation axis of the pendulum as the coordinate origin, and the system energy is 0 when the pendulum is in the inverted position, the potential energy of the pendulum can be expressed as

$$
E_{P}=m_{2} g l_{2}\left(1-\cos \theta_{2}\right)
$$

System kinetic energy can be expressed as

$$
E_{k}=\frac{1}{2} J \dot{\theta}_{2}^{2}
$$

where $\mathrm{J}$ is the moment of inertia of the pendulum about its centroid. After the system is idealized, that is, the system ignores the frictional force, the nonlinear motion equation of the system can be obtained.

$$
J \ddot{\theta}_{2}-m_{2} g l_{2} \sin \theta_{2}-m_{2} u l_{2} \cos \theta_{2}=0
$$


When the pendulum is upright $\theta 2= \pm \pi$, the potential energy of the system is. The total potential energy of the pendulum is

$$
E=\frac{1}{2} J \dot{\theta}_{2}^{2}+m_{2} g l_{2}\left(1-\cos \theta_{2}\right)
$$

For (6) to find the differential can be obtained:

Combine (5) with (7)

$$
\dot{E}=\dot{\theta}_{2}\left(J \dot{\theta}_{2}^{2}+m_{2} g l_{2} \sin \theta_{2}\right)
$$

$$
\dot{E}=m_{2} u l_{2} \dot{\theta}_{2} \cos \theta_{2}
$$

According to the axial acceleration proportional to the drive motor current, the shaft acceleration is proportional to the drive motor voltage, and the energy of the inverted pendulum is controlled by controlling the drive motor voltage [7-8].

Constructing Lyapunov function based on Lyapunov's theorem

Then the derivative is available

$$
V=\frac{1}{2}\left(E_{r}-E\right)^{2}
$$
\[ \frac{d V}{d t}=-\left(E_{r}-E\right) \frac{d E}{d t} \]
According to the Lyapunov theorem, the system is stable when the equation satisfies $V>0, \frac{d V}{d t}<0$.
Bring (8) into (10)

$$
\frac{d V}{d t}=-m_{2} u l_{2} \dot{\theta}_{2} \cos \theta_{2}\left(E_{r}-E\right)
$$

In order to satisfy the Lyapunov stability theorem, the controller is constructed.

$V>0, \frac{d V}{d t}<0 \quad u_{2}=k\left(E_{r}-E\right) \dot{\theta}_{2} \cos \theta_{2}$

When $\mathrm{k}>0$, Equation $V>0, \frac{d V}{d t}<0$ satisfies the Lyapunov theorem. According to equations (9), (11), and (12), in order to ensure that the system energy is continuously increased, the direction in which the connecting rod drives the motor to rotate is determined by the swing rod energy $\left(E_{r}-E\right)$, the swing $\operatorname{rod}$ angle $\theta_{2}$, and the swing rod angular acceleration .In order to keep the inverted pendulum swing up in a short time, the external force of the connecting rod can be expressed as

$$
u_{2}=u_{\max } \cdot \operatorname{sign}\left[\left(E_{r}-E\right) \dot{\theta}_{2} \cos \theta_{2}\right]
$$

Among them, $u_{\max }$ is the maximum acceleration of the connecting rod, and $\operatorname{sign}(\cdot)$ is the symbol function.

$$
\operatorname{sign}(x)=\left\{\begin{array}{lc}
1, & x>0 \\
0, & x=0 \\
-1, & x<0
\end{array}\right.
$$

The control law shown in equation (13) can shorten the swing-up time, but it would cause the inverted pendulum to shake. In order to make the swing-up process more stable, we adopt the following control strategy on the basis of equation (13).

$$
u_{2}=\operatorname{sat}_{u_{\max }}\left[\mu\left(E_{r}-E\right) \operatorname{sign}\left(\dot{\theta}_{2} \cos \theta_{2}\right)\right]
$$

Among them, is the adjustable control gain, and is the linear function with the saturation value .

During the physical experiment of the energy swing, the rotation of the connecting rod does not exceed the maximum acceleration to ensure that the motor will not be damaged, and the problem of the dead zone of the connecting rod needs to be considered. Due to the restriction of the connecting line below the connecting rod, there is a dead zone in the swing angle of the connecting rod.

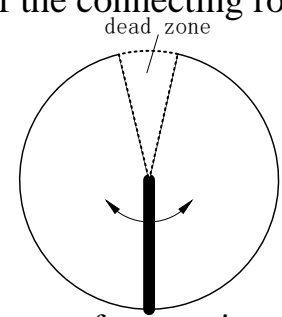

Fig. 3: Schematic diagram of connecting rod rotation dead zone. 
Considering the dead zone of the actual motion of the connecting rod, it is stipulated that the position of the connecting rod shown in Figure 3 is the zero point of the system coordinate, and the control law is further reformed.

$$
u_{2}=\operatorname{sign}\left(\theta_{2}\right) \lg \left[1-\frac{\left|\theta_{2}\right|}{5 / 6 \pi}\right]
$$

In order to maximize the protection of the experimental equipment, we extend the dead zone to $60^{\circ}$. When the link moves to around $150^{\circ}$, equation (16) produces an infinite repulsive force in the direction of $0^{\circ}$, and the resistance link approaches the dead zone to prevent equipment damage. According to the formula (15), (16), the final energy swing control law is obtained.

$$
u_{2}=\operatorname{sign}\left(\theta_{2}\right) \lg \left[1-\frac{\left|\theta_{2}\right|}{5 / 6 \pi}\right]+\operatorname{sat}_{u_{\max }}\left[\mu\left(E_{r}-E\right) \operatorname{sign}\left(\dot{\theta}_{2} \cos \theta_{2}\right)\right]
$$

Among them, $u_{\max }=6 \mathrm{~m} / \mathrm{s}^{2}$ is controllable gain, and is reference energy. According to the experiment, when the controllable gain is kept constant, the larger the reference energy $E_{r}$, the larger the amplitude of the control signal. Input voltage as shown in Figure 4.

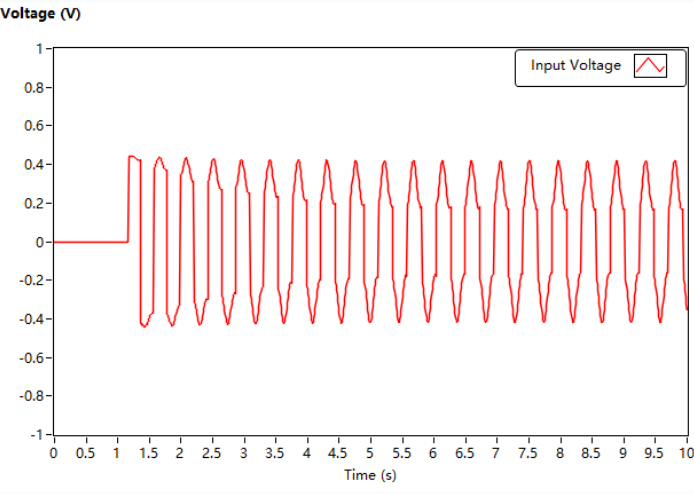

a. $\mu=50 \mathrm{Er}=5$

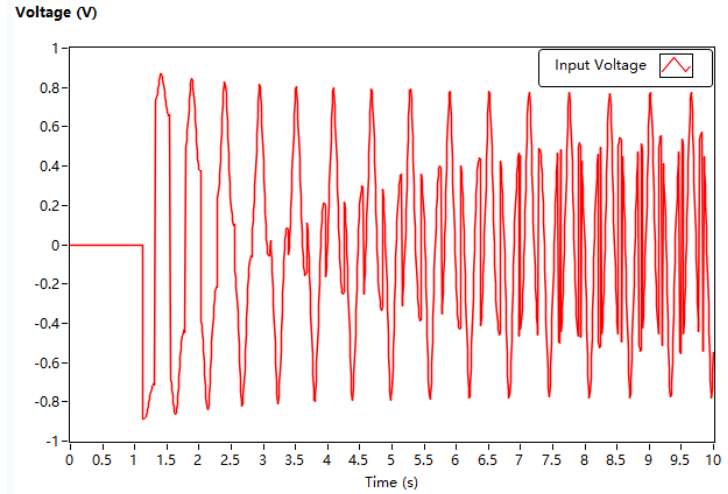

b. $\mu=50 \mathrm{Er}=10$

Fig. 4: When the size of Er is changed without changing $\mu$, the system input voltage comparison chart.

\subsection{Stabilization control based on LQR controller}

After the swing is completed, the LQR controller is used to realize the steady pendulum process. To do this, we linearize the system nonlinear model at $\left(\theta_{1}, \theta_{2}, \dot{\theta}_{1}, \dot{\theta}_{2}\right)=(0,0,0,0)$ the equilibrium point. Let , take the state variable $X=\left[\begin{array}{llll}\theta_{1} & \theta_{2} & \dot{\theta}_{1} & \dot{\theta}_{2}\end{array}\right]^{T}$, the output is, and the system linearization state space equation is $Y=\left[\begin{array}{ll}\theta_{1} & \theta_{2}\end{array}\right]^{T}$, and the system linearization state space equation is

$$
\begin{aligned}
& X=A X+B Y \\
& Y=C X+D u
\end{aligned}
$$

Bring data into

$$
\begin{aligned}
A= & \begin{array}{cccc}
0 & 0 & 1 & 0 \\
0 & 0 & 1 \\
0 & \frac{2 m_{2}^{2} g l_{1} l_{1}^{2}}{4 m_{1} l_{1}^{2} l_{2}^{2}-J_{1} J_{2}-J_{1} m_{2} l_{2}^{2}-J_{2} m_{1} l_{1}^{2}-m_{1} m_{2} l_{1}^{2} l_{2}^{2}} & 0 & 0 \\
0 & \frac{-m_{2} g l_{2}\left(J_{1}+m_{1} l_{1}^{2}\right)}{J_{1} J_{2}+J_{2} m_{1} l_{1}+J_{1} m_{2} l_{2}^{2}-4 m_{2} l_{1}^{2} l_{2}^{2}} & 0 & 0
\end{array} \mid=\left[\begin{array}{cccc}
0 & 0 & 1 & 0 \\
0 & 0 & 0 & 1 \\
0 & 174.49 & 0 & 0 \\
0 & 57.452 & 0 & 0
\end{array}\right] B=\left[\begin{array}{c}
0 \\
\frac{J_{2}+m_{2} l_{2}^{2}}{J_{1} J_{2}+J_{2} m_{1} l_{1}^{2}+J_{1} m_{2} l_{2}^{2}+m_{1} m_{2} l_{1}^{2} l_{2}^{2}-4 m_{2} l_{1}^{2} l_{2}^{2}} \\
\frac{2 m_{2} l_{1} l_{2}}{4 m_{2} l_{1}^{2} l_{2}^{2}-J_{1} J_{2}-J_{2} m_{1} l_{1}^{2}-J_{1} m_{2} l_{2}^{2}-m_{1} m_{2} l_{1}^{2} l_{2}^{2}}
\end{array}\right]=\left[\begin{array}{c}
0 \\
0 \\
54.51208 \\
57.45238
\end{array}\right], \\
C & =\left\lfloor\begin{array}{llll}
1 & 0 & 0 & 0 \\
0 & 1 & 0 & 0
\end{array}\right], D=\left\lfloor\begin{array}{l}
0 \\
0
\end{array}\right] .
\end{aligned}
$$

Known two performance index functions

$$
J=\frac{1}{2} \int_{0}^{\infty}\left(X^{T} Q X+U^{T} R U\right) d t
$$


where $\mathrm{X}$ is an $\mathrm{n}$-dimensional state variable, $\mathrm{Y}$ is an $\mathrm{m}$-dimensional output variable, $\mathrm{U}$ is an $\mathrm{r}$-dimensional input variable, and $\mathrm{Q}$ and $\mathrm{R}$ are weighting matrices. $\mathrm{Q}$ and $\mathrm{R}$ are used to balance the weights of $\mathrm{U}$ and $\mathrm{X}$. $\mathrm{R}$ is a positive definite matrix. When $\mathrm{R}>0, \mathrm{Q}$ is a semi positive definite matrix.

According to the optimal control theorem, after disturbing the equilibrium state, the system can quickly return to the equilibrium position $U^{*}(T)$ while keeping $\mathrm{J}$ minimum. Therefore, the optimal control law of the system is

$$
U^{*}=-R^{-1} B^{T} P X=-K X
$$

Among them, $P$ is the solution of Riccati equation

$$
A^{T} P+P A-P B R^{-1} B^{T} P+Q=0
$$

$K$ is a linear feedback matrix. Simultaneous equations (19) and (15) can get $P$ and feedback matrix $K$

$$
K=R^{-1} B^{T} P=\operatorname{lqr}(A, B, Q, R)
$$

According to the basic principle of trial-and-error method,

when the weighting matrix $Q=\left|\begin{array}{llll}1 & 0 & 0 & 0 \\ 0 & 1 & 0 & 0 \\ 0 & 0 & 1 & 0 \\ 0 & 0 & 0 & 0\end{array}\right|$ and $R=0.1$, the system meets the experimental requirements. Find the feedback matrix $K$

$$
K=\left[\begin{array}{llll}
-1 & 33.3499 & -1.21707 & 2.89486
\end{array}\right]
$$

\section{Experiment}

In order to verify the effectiveness of the control method in this paper, the single inverted pendulum produced by Canada Quanser Company was used in the physical experiment through LabVIEW experimental platform. Fig.5(a) is the result of the automatic swing control of the inverted pendulum. The blue curve indicates the swing angle of the connecting rod, the red curve indicates the swing angle of the swing rod, and the green curve indicates the energy variation curve of the swing rod.Figure 5(b) is the output voltage variation curve during the experiment.

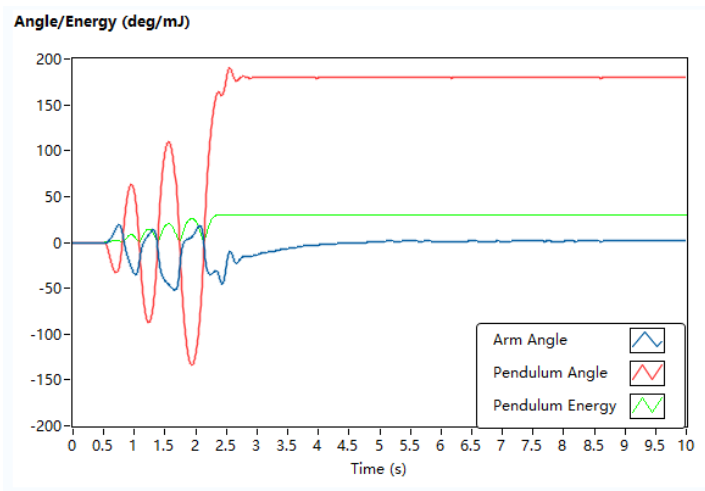

(a)

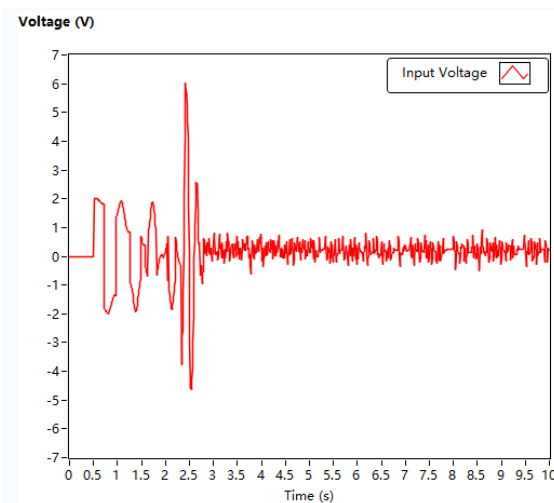

(b)

Fig. 5: (a)Experimental result of the automatic swing control of the inverted pendulum.

(b) Output voltage variation curve.

\section{References}

[1] Wang Laizhi, Yang Yuqiang. Research and Implementation of a Class of Rotating Inverted Pendulum and Its Control Device[J]. Journal of Southwest China Normal University(Natural Science), 2016, 41(08): 145-150.

[2] Jia Xinle, Zhang Xian library. Inverted pendulum piecewise control method [J]. Control engineering, 2016,23 (S1): 6-12. 
[3] Jian Haiyun, Du Zhenhua, Zou Ningbo, Shi Mingjiang. LQR controller design of inverted pendulum based on multipopulation genetic algorithm[J]. Control engineering, 2014, 21 (03): 391-394.

[4] Li Qiuju,Liu Kaian. Displacement control of single inverted pendulum based on generalized extended linearization[J]. Journal of North China Institute of Water Conservancy and Hydropower, 2011, 32(05): 95-98.

[5] Hu Lingyan, Liu Guoping, Liu Xiaoping, Zhang Hua. Simulation and Real-time Control of Inverted Pendulum System Based on Linear Quadratic Optimal Algorithm[J]. Mechanical Design and Manufacture, 2010 (01): 89-91.

[6] Wang Wei, Ren Xia. Optimal control of inverted pendulum system based on exact linearization theory[J]. Journal of Hefei University of Technology (Natural Science Edition), 2009,32(04): 500-502+507. 